\title{
The Singular Solutions of a Certain Differential Equation of the Second Order.
}

\section{By Mr Hugh Mitchell.}

The subject of the Singular Solutions of Differential Equations of higher orders than the first is not touched in the ordinary text. books. Their existence, for instance, is not mentioned by Forsyth in his Treatise. This is probably due to the fact that, while in the case of equations of the first order a theory has been developed by Cayley and others which connects the singular solution in a geometrical manner with the ordinary solutions (the singular solution being, of course, the envelope of the ordinary solutions), in the case of equations of, say, the second order no corresponding theory exists-at any rate, no corresponding theory has yet been developed. Our only guide in the subject at present is Cauchy's Existence Theorem, which points out where we are to look for singular solutions.

As few illustrations of the subject have been given, Professor Chrystal thought that the following one might be of interest and not unworthy of your notice.

The equation whose singular solutions are to be examined is one which is given by Forsyth in his treatise, chap IV. Miscell. Ex. 1 (IX.)

$$
\left(y^{\prime 2}-y y^{\prime \prime}\right)^{2}=n^{2}\left(y^{2}+a y^{\prime \prime 2}\right) \quad-\quad-\quad-
$$

This equation does not contain the independent variable $x$, so writing in the usual manner $y^{\prime} \frac{d y^{\prime}}{d y}$ for $y^{\prime \prime}$

$$
\begin{aligned}
& \text { we get } \quad y^{\prime 2}\left\{\left(y^{\prime}-y \frac{d y^{\prime}}{d y}\right)^{2}-n^{2}\left(1+a^{2}\left(\frac{d y^{\prime}}{d y}\right)^{2}\right)\right\}=0 \\
& \text { or } \quad y^{\prime}-y \frac{d y^{\prime}}{d y}= \pm n\left(1+a^{2}\left(\frac{d y^{\prime}}{d y}\right)^{2}\right)^{\frac{1}{2}}-
\end{aligned}
$$

This is Clairaut's form if we regard $y^{\prime}$ as dependent and $y$ as independent variable. Hence a first integral is

$$
y^{\prime}-y \mathbf{A}= \pm n\left(1+a^{2} \mathbf{A}^{2}\right)^{\frac{1}{2}}
$$


Integrating the linear equation we have

$$
y= \pm \frac{n}{\mathrm{~A}}\left(1+a^{2} \mathrm{~A}^{2}\right)^{\frac{1}{3}}+\mathrm{B} e^{\mathrm{Ax}}
$$

This is the ordinary complete primitive of the equation containing two arbitrary constants.

But since (2) is Clairaut's form, it has also a singular first integral, namely, its discriminant with regard to $\frac{d y^{\prime}}{d y}$

The integral of this is

$$
a^{2} y^{\prime 2}-a^{2} n^{2}+y^{2}=0
$$

$$
y=n a \sin \left(\frac{n}{a}+\mathrm{C}\right) \quad-\quad-
$$

Before going further, let us see what these two solutions correspond to geometrically.

The first is simply a twofold family of graphs of the exponential function, being always of the form $y=S+e^{T x}$. When $A$ has a definite value and $B$ varies, the curves form spreads issuing from the ends of the lines $y= \pm \frac{n}{\mathrm{~A}}\left(1+a^{2} \mathrm{~A}^{2}\right)^{\frac{1}{2}}$.

The axis of the spread is itself a solution, and corresponds to $B=0$. If we vary $A$ we vary the position of the axis of the spread. When $\mathrm{A}=\infty$ the axis is $y=n a$, when $\mathrm{A}=0$ the axis is $y=\infty$. Thus the axis of the spread of curves never lies between $y= \pm n a$.

The second solution (II.) is simply a one-fold family of curves of sines lying between the two lines $y= \pm n a$.

Now the solution (I.) contains two arbitrary constants, and is therefore the complete primitive and the solution whose existence for non-critical points is guaranteed by Cauchy's Existence Theorem. The solution (II.) is not a particular case of the first, and in fact has no member in common with it. It is therefore a singular solution.

In contrast with the theory of singular solutions of equations of the first order, two things are to be noticed. Firstly, that there is not merely one singular solution, but a one-fold infinity of singular solutions; and, secondiy, that the singular solutions cannot, of course, be regarded as envelopes of the ordinary solutions. 
The existence of this family of singular solutions was suggested by the ordinary process of solving the equation. But we may look at the point of view of Cauchy's Existence Theorem, and examine the various critical loci.

Arranging the equation in powers of the highest differential coefficient $y^{\prime \prime}$ the equation is of the form

$$
\begin{gathered}
y^{\prime \prime 2}\left(y^{2}-n^{2} a^{2}\right)-2 y y^{\prime 2} y^{\prime \prime}+y^{\prime 2}\left(y^{\prime 2}-n^{2}\right) \\
\mathrm{P} y^{\prime \prime 2}+\mathrm{Q} y^{\prime \prime}+\mathrm{R}=0
\end{gathered}
$$

and the critical loci are the discriminant with respect to $y^{\prime \prime}$ and the loci which make the coefficients $P, Q$ and $R$ vanish.

The $y^{\prime \prime}$-discriminant is

$$
y^{\prime \prime}\left(y^{\prime 2} a^{2}+y^{2}-n^{2} a^{2}\right)=0 .
$$

The factor in brackets is (3) which gave the solution (II.)

$$
y^{\prime 2}=0 \text { gives } y=\mathrm{D}
$$

which on trial is found to be a solution of the equation, and is thus a second family of singular solutions. It is to be noticed, however, that outside of the two lines $y= \pm n a$ its members are also members of the complete primitive. But part of the family is not included in the complete primitive, and since it consists of elements $\left(x, y, y^{\prime}\right)$ for which Cauchy's normal form ceases to be synectic, all the members of the family may be considered singular solutions.

We next examine the locus $R=0$

$$
\begin{array}{ll}
\text { that is } & y^{\prime 2}-n^{2}=0 \\
\text { that is } & y= \pm n x+\mathrm{E}
\end{array}
$$

This on trial is found to satisfy the equation, and is thus a third family of singular solutions.

There remains to be considered the locus $\mathbf{P}=0$

$$
\text { or } y^{2}-n^{2} a^{2}=0 \text {. }
$$

This locus might have come in otherwise. It is, in fact, the envelope singular solution of (3), which was itself a singular first integral of the equation. It is therefore a solution of the equation. It is, however, a limiting form of the complete primitive as well.

This example shows in a remarkable manner how far from being the only solution of a differential equation the complete primitive 
containing the full number of arbitrary constants may be. The critical loci, in fact, will be in general differential equations of an order lower by one than the differential equation itself, and they may furnish singular solutions containing that number of arbitrary constants.

In the case of equations of the first order, the critical locus has been analysed into cusp-locus, tac-locus, and envelope singular solution, and criteria for each have been given. No such analysis of the critical locus of an equation of, say, the second order has been attempted, and no criterion for the existence of singular solutions given. Still, it is possible that in a physical problem it might be well to bear in mind that, outside of the most general solution of an equation, solutions of a less degree of generality might also exist, and might be of a very different form. 